\title{
Review
}

\section{Host-Parasite Relation in Invasive Aspergillosis}

\author{
Andreas Schaffner \\ Department of Medicine, University Hospital, Zürich
}

Invasive aspergillosis has become one of the most important infectious complications of intensive modern medicine often limiting the success of oncological treatments and transplantation. The rationale for this is found in the effects of immunosuppressive therapies and to a lesser degree underlying disease processes as well as in properties of the fungus. The double pronged nature of host defenses directed against spores and hyphae of Aspergilli is affected by glucocorticoids and myeloablative therapy. Resident alveolar macrophages are the major players eliminating inhaled conidia. Neutrophils, efficiently killing hyphae. These defense mechanisms are so solid that even after the inhalation of billions of spores infection is reliably prevented in man. Mechanisms by which glucocorticoids prevent macrophages from first inhibiting germination of ingested conidia and then killing them have not been elucidated. Mobilization of neutrophils is also hampered by glucocorticoids that have the potential to suppress the expression of an array of neutrophil chemokines by macrophages. Glucocorticoids are thus able to abrogate defenses against Aspergilli on their own, affecting both lines of defense. In neutrophil granulocytes oxidative killing systems directed against hyphae are of major importance as pointed out by infections in children with chronic granulomatous disease, but other killing systems such as defensins and possibly thrombocidins are also of importance. To date it remains speculative that platelet derived thrombocidins play an important role, but such speculations are tempting in view of the angio-invasive nature of the fungus and the coexisting thrombocytopenia in many patients with aspergillosis. 